\title{
Tumor estromal gastrointestinal
}

\section{Gastrointestinal stromal tumor}

\author{
Rolando Mendívil ${ }^{1 \mathrm{a}, 2 \mathrm{~b}}$, José Luis Cabanillas ${ }^{2 c, 3 a}$, Noris Lozano ${ }^{2 c}$, Mario De la Cruz ${ }^{2 c}$, \\ Rocío Mendívil ${ }^{4 \mathrm{~d}}$ \\ ${ }^{1}$ Servicio de Cirugía H3, Hospital Nacional Dos de Mayo. Lima, Perú. \\ 2 Facultad de Medicina, Universidad Nacional Mayor de San Marcos. Lima, Perú. \\ ${ }^{3}$ Servicio de Patología, Hospital Nacional Dos de Mayo. Lima, Perú. \\ ${ }^{4}$ Corporación Sanitaria Parc Tauli. Barcelona, España. \\ a Médico Asistente; ${ }^{b}$ Profesor Principal; c Médico Cirujano; ${ }^{d}$ Médico Residente
}

\section{Resumen}

Los tumores estromales gastrointestinales son los tumores mesenquimáticos más frecuentes. Se localizan con frecuencia en el estómago y sus manifestaciones clínicas son variadas. Presentamos el caso de una paciente de 57 años que ingresó por hemorragia digestiva de dos días de evolución. Los estudios de imagen demostraron un tumor en pared gástrica. La paciente fue sometida a resección quirúrgica del tumor y el diagnóstico definitivo se realizó utilizando marcadores CD117 y CD34. A pesar de que el tratamiento definitivo es la resección quirúrgica, se ha desarrollado nuevos modelos de tratamiento utilizando terapia molecular dirigida. Diversos estudios están llevándose a cabo en la actualidad y algunos muestran resultados prometedores.

Palabras clave: Tumores estromales gastrointestinales; inmunohistoquímica; antigens, CD117; antígenos CD34.

\begin{abstract}
Gastrointestinal stromal tumors are the most frequent mesenchimal neoplasms of gastrointestinal tract. They arise more frequently in the stomach and have a broad clinical spectrum. We report the case of a fifty-seven year-old female with history of two day gastrointestinal bleeding. The patient underwent surgical resection and the diagnosis was done using CD117 and CD34 markers. Though surgical resection is the only definitive treatment new therapeutic models are in development that use molecular targeted drug therapy. Various clinical essays are currently in course and some of them show promising results.

Key words: Gastrointestinal stromal tumors; immunohistochemistry; antigens, CD117; antigens, CD34.
\end{abstract}

\section{INTRODUCCIÓN}

Los tumores estromales gastrointestinales (TEG) son tumores mesenquimáticos del tracto gastrointestinal ${ }^{(1)}$, histológicamente derivados de las células intersticiales de Cajal o de las células madre que originan a dichas células ${ }^{(2,3)}$.

Los TEG constituyen los tumores mesenquimatosos más frecuentes del tubo digestivo, aunque representan 0,1 a $3 \%{ }^{(3)}$ de las neoplasias gastrointestinales, luego del adenocarcinoma y el linfoma ${ }^{(4)}$.

Un 60\% de los TEG se ubican en el estómago, 30\% en el intestino delgado, $5 \%$ en esófago y $5 \%$ en otras localizaciones ${ }^{(5)}$. Se presentan con mayor frecuencia entre la quinta y la sétima décadas de la vida, siendo mayor su incidencia en la sexta década, sin ninguna predilección en cuanto al género (2).

Entre 30 y $50 \%$ de los portadores de un TEG son asintomáticos al momento del diagnóstico ${ }^{(6,7)}$, principalmente cuando la neoplasia es de pequeño tamaño. Clínicamente pueden producir dolor abdominal mal definido, saciedad precoz, ictericia, disfagia, fiebre y otros ${ }^{(5)}$; además, suelen ser causa de sangrado digestivo importante ${ }^{(8)}$.

El diagnóstico generalmente se puede sospechar cuando existe historia de sangrado gastrointestinal asociado a una lesión tumoral en la pared gastrointestinal, evidenciada por estudios de imágenes ${ }^{(9)}$.

El tratamiento de los TEG es quirúrgico y el tipo de operación depende del tamaño y ubicación del tumor, la gastrectomía total para los tumores del tercio superior del estómago y la subtotal para los del cuerpo y antro ${ }^{(10)}$.

En los pacientes con tumor recidivante o en quienes el tumor primario o la enfermedad metastásica es irresecable, se puede utilizar mesilato de imatinib, fármaco inhibidor del crecimiento tumoral, como tratamiento adyuvante ${ }^{(11)}$.

El objetivo del presente artículo es dar a conocer el presente caso, porque a pesar de su incidencia baja es una causa importante de morbilidad y porque no se ha publicado muchos casos en nuestro medio.

\section{CASO CLÍNICO}

Paciente mujer de 57 años que ingresó por el servicio de Emergencia del Hospital Nacional Dos de Mayo con historia de epigastralgia, melena, palidez y fatiga de dos días de evolución; en dicho ingreso, se le administró sangre. La paciente no tenía historia de sangrado previo. No había antecedente de uso de antiinflamatorios no esteroideos, ni historia de consumo de alcohol u otras sustancias tóxicas. Tampoco tenía antecedente de enfermedad hepática. Hacía 8 años había sido sometida a endoscopia digestiva por síntomas de gastritis, la que encontró una lesión elevada Yamada 1 en cuerpo del estómago asociado a gastritis erosiva moderada. Se le realizó una biopsia para descartar leiomioma. El estudio anatomopatológico concluyó gastritis crónica activa con presencia de Helicobacter pylori $(3+)$ y áreas de metaplasia intestinal incompleta. Recibió tratamiento para gastritis y para erradicar al H. pylori. La paciente negó tener antecedente familiar de cáncer gastrointestinal.

Al examen físico, se encontraba en aparente regular estado general; despierta 
y orientada. La piel y mucosas estaban moderadamente pálidas. No había ictericia ni adenopatías cervicales, axilares ni inguinales. En el examen de tórax, pulmones y aparato cardiovascular no hubo hallazgos significativos. En el abdomen, había distensión leve y sin circulación colateral. A la palpación, se encontró una tumoración en epigastrio, móvil, no dolorosa. Los ruidos hidroaéreos eran normales. No hubo otros hallazgos significativos en el examen físico.

Los exámenes de laboratorio estaban dentro de los rangos normales, a excepción de la hemoglobina baja. La ecografía abdominal no tuvo hallazgos significativos. La radiografía de tórax no evidenció ensanchamiento del mediastino y los campos pulmonares eran radiolúcidos. Los senos costofrénicos se encontraban libres. La endoscopia digestiva alta evidenció una lesión elevada submucosa de bordes definidos de $80 \times 100 \mathrm{~mm}$ con áreas hipodensas difusas, con efecto de tumoración. La tomografía computarizada evidenció un tumor de pared en el cuerpo del estómago que no comprometía la mucosa, como se puede observar en la figura 1 . No se evidenció alguna lesión hepática.

El hallazgo intraoperatorio fue una tumoración gástrica submucosa de $15 \times 12 \times 10 \mathrm{~cm}$ ubicada en la pared poste- rior de antro y cuerpo. Se observaba dos zonas diferenciadas: una zona de aspecto sarcomatoide, que protruía hacia la luz desplazando la mucosa, y otra de aspecto hemangiomatoso que se extendía hacia el exterior, irrumpiendo la serosa del estómago y adosándose al mesocolon a nivel de la pared posterior, entre el cuerpo y el antro. Se le realizó gastrectomía subtotal con gastroduodenostomía tipo Billroth I. No hubo evidencia de compromiso ganglionar.

En el estudio de anatomía patológica, se observó macroscópicamente un tumor sólido de aspecto polipoide sin compromiso de la mucosa y con extensión hacia la cara externa del estómago. Al corte, el tumor tenía localización intramural submucosa, bien delimitada, con superficie regular blanquecino-grisácea, con lesiones pseudoquísticas, zonas de necrosis, congestión y hemorragias focales, como muestra claramente la figura 2. Una región del tumor comprometía la serosa y se encontraba adherida al mesocolon.

Al examen microscópico con tinción hematoxilina eosina se observó una lesión tumoral submucosa con células fusiformes en empalizada y con vacuolización perinuclear (figura 3). Había actividad mitótica menor de 5 en 50 campos, con un grado moderado de atipia nuclear. El estudio inmunohistoquímico evidenció

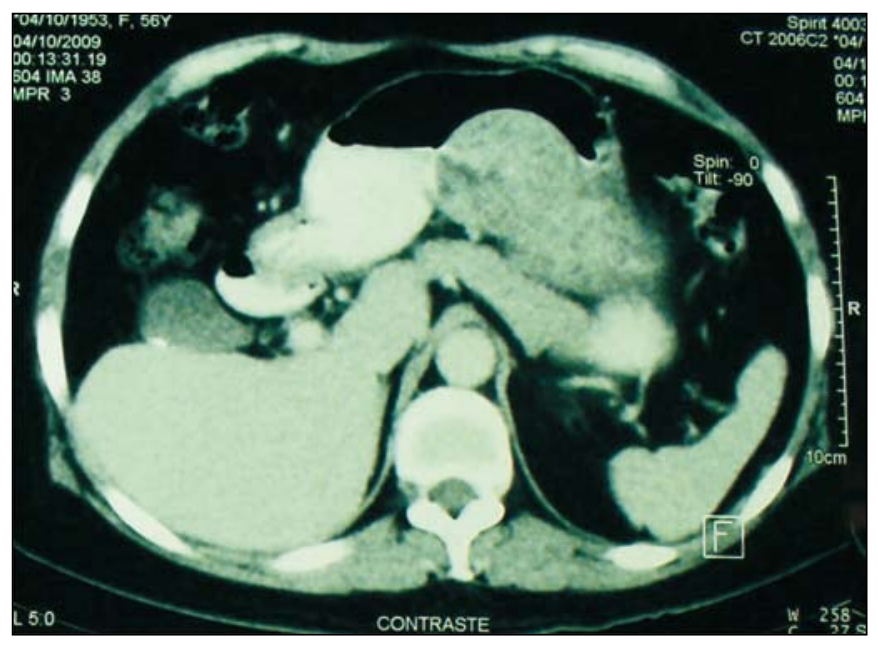

Figura 1. Tomografía computarizada contrastada que muestra una tumoración bien delimitada en la pared posterior del estómago, sin lesión evidente de la mucosa. Dentro de la tumoración se observa imágenes heterogéneas hipodensas. El parénquima hepático se encuentra sin lesiones evidentes. No se evidencia adenopatías retroperitoneales. fuerte positividad para CD117 y CD34. El tumor resultó ser también fuertemente positivo para vimentina y negativo para cromogranina (figura 4). El diagnóstico anatomopatológico fue tumor estromal gástrico, de grado intermedio de malignidad, con infiltración a serosa.

\section{DISCUSIÓN}

Los tumores estromales gastrointestinales son tumores mesenquimáticos del tracto gastrointestinal, histológicamente derivados de las células intersticiales de Cajal ${ }^{(1-3)}$, localizadas principalmente a nivel de los plexos mientéricos de Meissner, aunque se pueden encontrar también a nivel de la capa muscular lisa y por dentro de ella, en los plexos submucosos de Auberbach. Las células intersticiales de Cajal forman parte de una compleja red celular a lo largo del tubo digestivo y cumplen función de marcapaso del músculo liso gastrointestinal; contribuyen a facilitar la propagación de impulsos nerviosos y a mediar eventos de neurotransmisión que regulan la actividad peristáltica. Morfológicamente, podría decirse que presentan características compartidas de músculo liso y de célula nerviosa autónoma periférica ${ }^{(12)}$.

La paciente que presentamos ingresó al hospital con historia de hemorragia digestiva alta, fue hospitalizada y durante los estudios complementarios se detectó una lesión tumoral localizada en la pared gástrica, con lo cual se planteó dentro del diagnóstico diferencial la presencia probable de un TEG; sin embargo, entre los tumores del tracto gastrointestinal, los tumores no epiteliales tienen una incidencia que no excede el $4 \%$ de todos los tumores del estómago ${ }^{(13)}$. El estudio de Portanova y col, en el Hospital Edgardo Rebagliati Martins, se registra a los tumores estromales como la tercera causa de cáncer gástrico luego del adenocarcinoma y del linfoma ${ }^{(14)}$. A pesar de esto, el estómago es la localización más frecuente de los TEG (60 a 70\%), seguido del intestino delgado (20 a 30\%), colon y recto (5\%) y menos de $5 \%$ en el esófago y en localizaciones extragastrointestinales ${ }^{(15-17)}$.

Las manifestaciones clínicas dependen del tamaño y de la localización del tumor. 


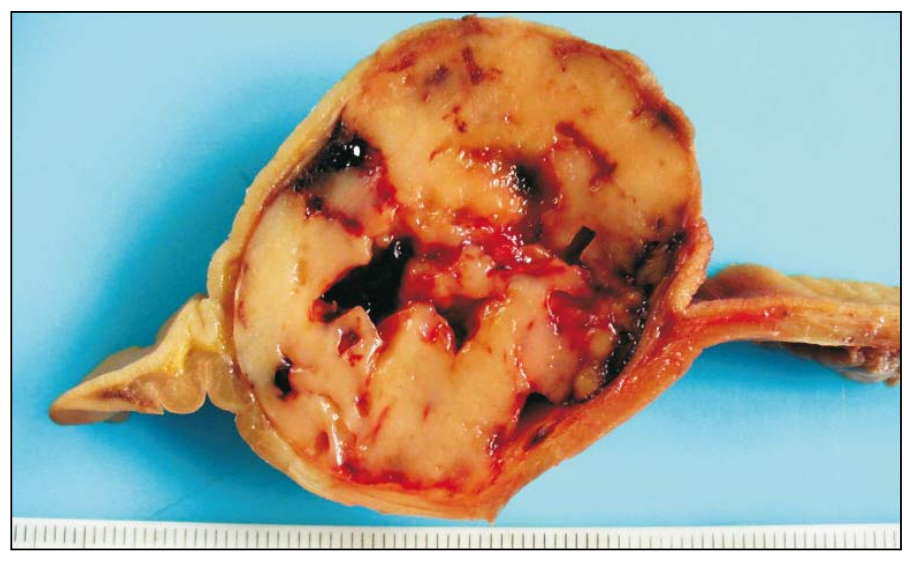

Figura 2. Tumor estromal que protruye hacia la luz gástrica y se extiende hacia la serosa. Se puede observar zonas de degeneración quística, con áreas de congestión, hemorragia y necrosis.

Los TEG pequeños son diagnosticados usualmente como un hallazgo durante una intervención quirúrgica o durante estudios de imágenes por otras causas. Los TEG sintomáticos se pueden presentar con disfagia, síntomas gástricos inespecíficos, dolor abdominal y hemorragia digestiva alta o baja ${ }^{(15)}$. Las manifestaciones clínicas de sangrado digestivo pueden estar presentes en la cuarta parte de los pacientes con un TEG ${ }^{(5)}$. Nuestra paciente tenía diagnóstico de gastritis crónica desde hace 8 años y había recibido tratamiento para erradicación de H. pylori. En esta ocasión ingresó por hemorragia digestiva alta de dos días de evolución que la llevó a un estado de anemia aguda.

La valoración inicial de un TEG es similar a la evaluación de otros tumores gastrointestinales. La radiografía abdominal a doble contraste puede demostrar defectos por sustracción, característicamente lisas, lineales y de bordes definidos. Al estudio endoscópico, los TEG se pueden mostrar como una tumoración que protruye de la pared gastrointestinal y la mucosa puede tener signos de ulceración o sangrado. La tomografía computarizada y la resonancia magnética nuclear son esenciales en la evaluación de la extensión del tumor y la presencia de metástasis ${ }^{(5)}$. Nuestra paciente había tenido estudios endoscópicos previos, en uno de los cuales se observó una lesión elevada Yamada 1 cuyo estudio resultó negativo para leiomioma. La endoscopia que se le realizó en esta hospitalización, el estudio contrastado del tubo digestivo y la tomografía computarizada evidenciaron

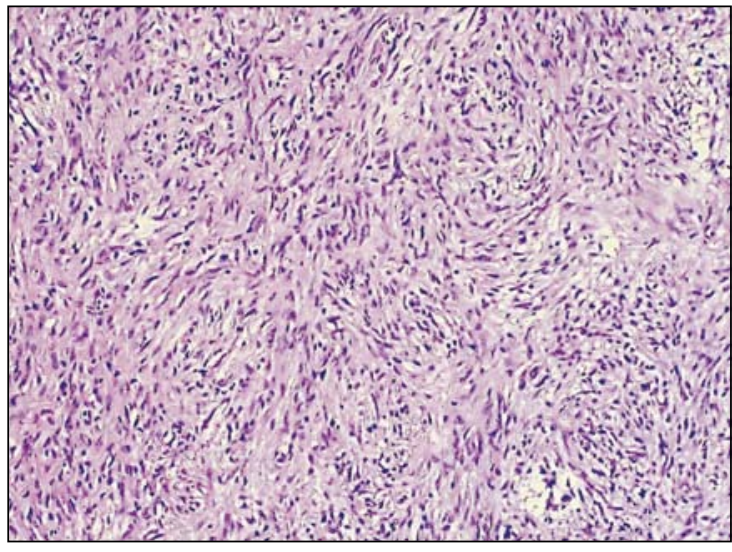

Figura 3. Corte histológico en el que se observa abundantes células fusiformes con vacuolización perinuclear. Tinción HE, 20x. una tumoración en la pared gástrica de crecimiento submucoso.

Macroscópicamente, las dimensiones de los TEG varían desde unos milímetros a decenas de centímetros, alcanzando solo el 20\% de ellos un tamaño superior a los $10 \mathrm{~cm}$. En nuestra paciente, el tumor había alcanzado un diámetro mayor de $15 \mathrm{~cm}$ y había comprometido la serosa y el mesocolon. Al corte presentaba superficie lisa y fibrosa, degeneración quística y zonas necróticas y hemorrágicas, características típicas de este tipo de tumores (4,5). Microscópicamente, la morfología celular era de tipo fusiforme, lo que ocurre en $77 \%$ de los casos.

En la actualidad, el diagnóstico y estadificación de estos tumores se realiza por estudio ultraestructural e inmunohistoquímico ${ }^{(18)}$, ya que desde el punto de vista histológico se debe hacer un diagnóstico diferencial con otros tumores no epiteliales del tracto gastrointestinal, como tumores del músculo liso, tumores desmoides, schwannomas, tumores fibrosos solitarios, pólipos inflamatorios, tumores metastásicos y sarcomas primarios, como melanoma maligno, angiosarcoma y el liposarcoma ${ }^{(19,20)}$. Se ha determinado, con el uso de anticuerpos monoclonales, que aproximadamente $95 \%$ de los TEG expresan la proteína CD117, también denominada proteína KIT, mientras que la expresión de CD34 ocurre en 60 a 70\% de estos tumores.

Los TEG también pueden presentar positividad a marcadores expresados por otros tumores, como leiomiomas, leiomiosarcomas o schwannomas. Aproximadamente $30 \%$ de los TEG son positivos para actina de músculo liso (SMA) y un porcentaje menor lo es para desmina. También pueden resultar positivos para nestina y vimentina, aunque son negativos para la proteína acídica fibrilar glial, lo que los diferencia de los schwannomas ${ }^{(20-23)}$. La muestra tomada en el presente caso resultó fuertemente positiva para CD117, CD34 y vimentina, con lo cual se confirmó el diagnóstico de TEG.

Aunque el comportamiento de los TEG es difícil de predecir, el tamaño es uno de los criterios cardinales para determinar el grado de malignidad del tumor, además del número de mitosis ${ }^{(24,25)}$. Shiu 


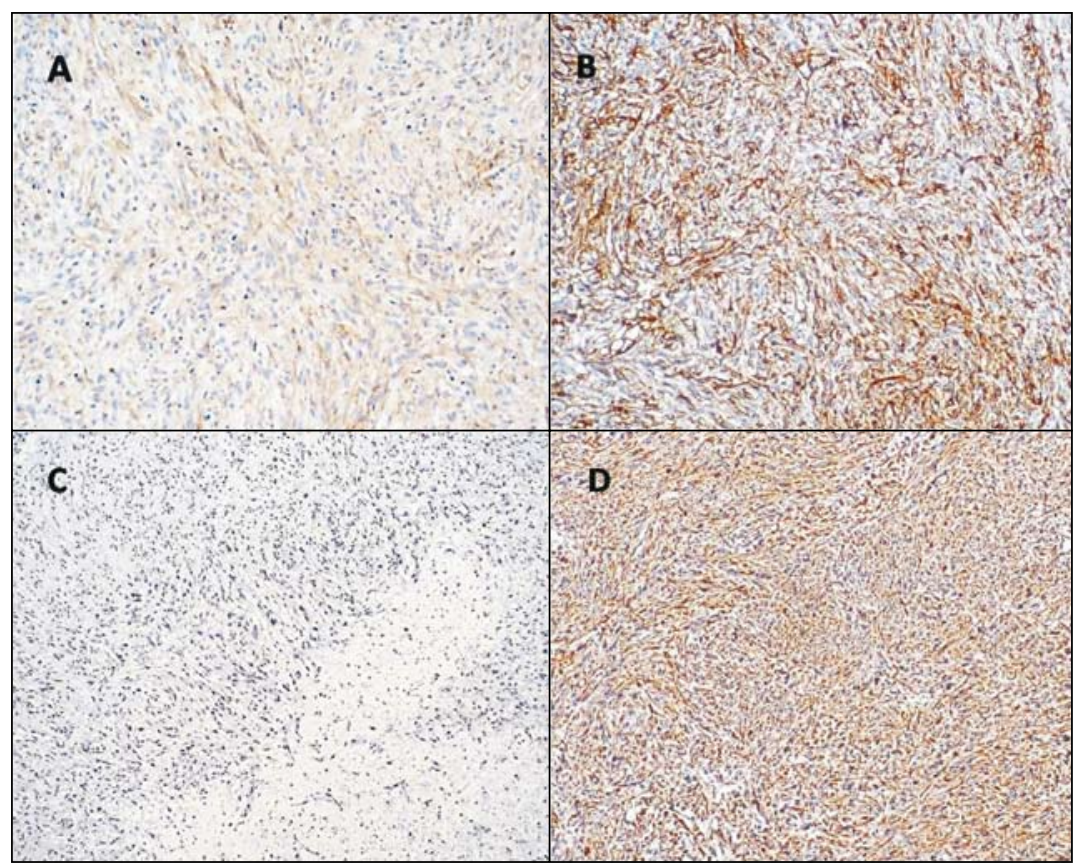

Figura 4. El análisis imunohistoquímico muestra CD117+ (A), CD34+ (B), cromogranina- (C) y vimentina+ (D). Tinciones HE contrastadas, 20x. y col comunicaron que los tumores menores de $5 \mathrm{~cm}$ nunca daban metástasis, 15 a 30\% de los que medían entre 6 y 10 cm daban metástasis y los mayores de 10 cm daban metástasis en $60 \%$ de los casos; concluyeron que los tumores mayores de $6 \mathrm{~cm}$ deberían ser considerados malignos, debido a su potencial metastásico ${ }^{(26)}$.

Los TEG metastatizan fundamentalmente por vía hematógena y el hígado es el sitio donde se observa con más frecuencia las metástasis. Con menor frecuencia hacen metástasis al peritoneo y pulmones, mientras que las metástasis linfáticas son excepcionales ${ }^{(27)}$. Se ha tratado de definir criterios para evaluar el potencial maligno de estos tumores ${ }^{(28)}$. En base a los resultados de diversos estudios se ha llegado a un consenso y se ha elaborado una tabla (tabla) para tratar de predecir el potencial metastásico o de recurrencia luego de la resección quirúrgica, que utiliza como criterios el índice mitótico y el tamaño del tumor ${ }^{(29)}$. El índice mitótico es el número de mitosis por 50 campos de gran aumento, vistas al microscopio de luz, y sería el factor predictor más importante. Miettinen y col encontraron que $86 \%$ de pacientes con índice mitótico de 5 o más y un tumor mayor de $10 \mathrm{~cm}$ presentaba metástasis al momento del diagnóstico ${ }^{(30)}$.
En nuestra paciente, no hubo evidencia de metástasis hepática en la tomografía computarizada. Tampoco se encontró compromiso ganglionar; sin embargo, el tamaño del tumor mayor de $10 \mathrm{~cm}$ supone una conducta agresiva y un riesgo alto de recidiva.

El tratamiento de los TEG es quirúrgico; el tipo de operación depende del tamaño y ubicación del tumor, la gastrectomía total para los tumores del tercio superior del estómago y la subtotal para los del cuerpo y antro ${ }^{(10)}$. En nuestra paciente se realizó una gastrectomía subtotal con reconstrucción tipo Billroth I, por la ubicación del tumor, con evolución postoperatoria satisfactoria, a pesar de una fístula de bajo débito que cerró espontáneamente a los tres días.

Se calcula que $50 \%$ de los pacientes a los que se les ha realizado resección completa tiene recidiva del tumor y la supervivencia a los 5 años es de $50 \%$ (31). Es discutido y está aún en estudio el uso de imatinib luego de una resección completa, en pacientes con enfermedad localizada, aunque algunos estudios han encontrado disminución en la tasa de recurrencia ${ }^{(32-34)}$. Sí se ha demostrado un aumento en la tasa de supervivencia en pacientes con tumores irresecables o metastásicos ${ }^{(35-37)}$. En nuestra paciente no hubo evidencia de metástasis; sin embargo, al tratarse de un tumor con características de alto riesgo se decidió iniciar el tratamiento con mesilato de imatinib, a la dosis recomendada de $400 \mathrm{mg}$ por día.

Podemos deducir que la edad de la paciente coincide en el rango de mayor frecuencia en que se presentan estos tumores, así como la hemorragia digestiva, que es una manifestación muy frecuente. Los estudios de imágenes permiten sospechar el diagnóstico; sin embargo, el diagnóstico definitivo se realiza con estudio inmunohistoquímico. El tamaño del tumor y las características microscópicas son factores que influyen de manera importante en el pronóstico de esta enfermedad.

La resección quirúrgica completa es curativa en $50 \%$ de los pacientes con enfermedad localizada. No existe consenso sobre el uso de imatinib en estos pacientes, aunque ha demostrado ser eficaz en pacientes con enfermedad metastásica o irresecable, por lo que se mantiene la
Tabla 1. Riesgo de conducta agresiva de los tumores estromales gastrointestinales, según el tamaño del tumor y el índice mitótico (Fletcher y col, 2002).

\begin{tabular}{lcc}
\hline & Tamaño (diámetro mayor) & Índice mitótico \\
\hline Riesgo muy bajo & $<2 \mathrm{~cm}$ & $<5 / 50$ campos \\
Riesgo bajo & $2-5 \mathrm{~cm}$ & $<5 / 50$ campos \\
Riesgo intermedio & $<5 \mathrm{~cm}$ & $6-10 / 50$ campos \\
& $5-10 \mathrm{~cm}$ & $<5 / 50$ campos \\
Riesgo alto & $>5 \mathrm{~cm}$ & $>5 / 50$ campos \\
& $>10 \mathrm{~cm}$ & Cualquiera \\
\hline
\end{tabular}


expectativa por los resultados de estudios multicéntricos que lleven al diseño de nuevas guías de tratamiento.

A la fecha, la paciente ha evolucionado satisfactoriamente.

\section{REFERENCIAS BIBLIOGRÁFICAS}

1. Alvarado-Cabrero I, Barreto R, Benítez H, Flores JP, Medrano Guzmán R, Torres S, et al. Lineamientos actualizados en el abordaje de tumores del estroma gastrointestinal (GIST) en México. GAMO. 2008;7(Supl 1):1-18.

2. Sircar K, Hewlett BR, Huizinga JD, Chorneyko K, Berezin I, Riddell RH. Interstitial cells of Cajal as precursors of gastrointestinal stromal tumors. Am J Surg Pathol. 1999;23(4):377-89.

3. Gómez S, Gómez C, Segura J. Tratamiento de los tumores de la estroma gastrointestinal (GIST). Med Clin (Barc). 2006;127(17):660-8.

4. Casali P, Jost L, Reichard P, Schlemmer M. On behalf of the ESMO Guidelines Working Group Gastrointestinal stromal tumors: ESMO Clinical recommendations for diagnosis, treatment and follow-up. Ann Oncol. 2008;19 Suppl 2:ii35-8.

5. Candelaria M, De la Garza J, Dueñas-González A. A clinical and biological overview of gastrointestinal stromal tumors. Med Oncol. 2005;22(1):1-10.

6. Palmiere B, Cogni P, Criscuolo M, Di Gregorio C. Leiomioblastoma dello stomaco: Ulteriore contributo casistico. Minerva Chir. 1991;46:561-6.

7. Sacco D, Paron L, Bardella R, Spidalieri G, Cardino L. Leiomiosarcoma gástrico. Osservazione di tre casi clinici in urgenza. Minerva Chir. 1994:49:995-9.

8. Benavides C, Burmeister R, Apablaza S, Garcia C, Pinedo M. Tumores gástricos estromales. Rev Chil Cir. 2002;54:44-8.

9. Bensimhon D, Soyer P, Boudiaf M, Fargeaudou Y, Nemeth J, Pocard M, et al. Imaging of gastrointestinal stromal tumors. J Radiol. 2009;90(4):469-80.

10. Connolly EM, Gaffney E, Reynolds JV. Gastrointestinal stromal tumors. Br J Surg. 2003;90:1178-86.

11. NCCN. Task Force Report: Optimal management of patients with gatrointestinal stromal tumor (GIST) - Update of the NCCN Clinical Practice Guidelines. The National Comprehensive Cancer Network. 2007;5 Suppl 2:1-31.

12. Kindblom LG, Remotti HE, Aldenborg F, MeisKindblom JM. Gastrointestinal pacemaker cell tumor (GIPACT): gastrointestinal stromal tumors show phenotypic characteristics of the interstitial cells of Cajal. Am J Pathol. 1998;152:1259-69.

13. Sanders L, Silverman M, Rossi R, Braasch J, Munson L. Gastric smooth muscle tumors: Diagnostic dilemmas and factors affecting outcome. World J Surg. 1996;20:992-5.
14. Portanova M, Vargas F, Lombardi E, Carbajal R, Palacios N, Rodríguez Cy col. Tratamiento quirúrgico del cáncer gástrico en un servicio especializado. Rev Gastroenterol Peru. 2005;25:239-47.

15. Miettinen M, Lasota J. Gastrointestinal stromal tumors - definition, clinical, histological, immunohistochemical, and molecular genetic features and differential diagnosis. Virchows Arch. 2001;438:1-12.

16. Jiménez M, Costa D, López-Andújar R, Mir J, Velasco JA. Retroperitoneal GIST: an unusual location for a rare tumour. Cir Esp. 2010;87(4):263-4.

17. Ceballos KM, Francis JA, Mazurka JL. Gastrointestinal stromal tumor presenting as a recurrent vaginal mass. Arch Pathol Lab Med. 2004;128(12):1442-4.

18. Domínguez J, Díaz J, Rodríguez L, Madrigal G, Queral QR, Collera S, et al. Tumor estromal intestinal. Rev Cubana Cir (revista en internet). 2008;47(2).Disponible en:http://scielo.sld.cu/ scielo.php?script=sci_arttext $\&$ pid $=$ S00347493200800020000\&lng=es.

19. Shidham V, Chivukula M, Gupta D, Rao RN, Komorowski R. Immunohistochemical comparison of gastrointestinal stromal tumor and solitary fibrous tumor. Arch Pathol Lab Med. 2002;126(10):118992.

20.Laurini JA, Carter JE. Gastrointestinal stromal tumors: a review of the literature. Arch Pathol Lab Med. 2010;134:134-41.

21. Miettinen M, Lasota J. Gastrointestinal stromal tumors: review on morphology, molecular pathology, prognosis, and differential diagnosis. Arch Pathol Lab Med. 2006;130:1466-78.

22. Wang L, Vargas H, French SW. Cellular origin of gastrointestinal stromal tumors. Arch Pathol Lab Med. 2000;124(10):1471-5.

23. Miettinen M, Sobin LH, Sarlomo-Rikala M. Immunohistochemical spectrum of GISTs at different sites and their differential diagnosis with a reference to CD117 (KIT). Mod Pathol. 2000;13(10):1134-42.

24. Davis GB, Blanchard DK, Hatch GF 3rd, WertheimerHatch L, Hatch KF, Foster RS Jr, et al. Tumors of the stomach. World I Surg. 2000;24:412-20.

25. Amin MB, Ma CK, Linden MD, Kubus JJ, Zarbo RJ. Prognostic value of proliferating cell nuclear antigen index in gastric stromal tumors. Am J Clin Pathol. 1993;100:428-32.

26. Shiu MH, Farr GH, Papachristou DN, Hajdu SI. Myosarcoma of the stomach: natural history, prognostic factors and management. Cancer. 1982;49:177-87.

27. Kwon SJ. Korean Gastric Cancer Study Group, Surgery and prognostic factors for gastric stromal tumor. World J Surg. 2001;25(3):290-5.

28. Lev D, Kariv Y, Issakov J, Merhav H, Berger E, Merimsky 0, et al. Gastrointestinal stromal sarcomas. Br J Surg. 1999;86:545-9.
29. Fletcher C, Berman J, Corless C, Gorstein F, Lasota J, Longlev B, et al. Diagnosis of gastrointestinal stromal tumors: A consensus approach. Hum Pathol. 2002;33:459-65.

30. Miettinem M, Sobin L, Lasota J. Gastrointestinal stromal tumors of the stomach. A clinicopathologic, inmunohistochemical, and molecular genetic study of 1765 cases with long-term follow-up. Am I Surg Pathol. 2005;29:52-68.

31. Poveda A, Maurel J, Martín J, Artigas V, Casado A, Cervra J y col. Guía de práctica clínica en los tumores estromales gastrointestinales: actualización 2008. Grupo Español de Investigación en Sarcomas (GEIS). Cir Esp. 2008;84(Supl 1):1-21.

32. Heger U, Weitz J, Lordick F. Indications for pre- and postoperative treatment with imatinib for gastrointestinal stromal tumors. Chirurg. 2008;79(7):630-7.

33. Dematteo RP, Ballman KV, Antonescu CR, Maki RG, Pisters PW, Demetri GD, et al. Adjuvant imatinib mesylate after resection of localised, primary gastrointestinal stromal tumour: a randomised, double-blind, placebo-controlled trial. Lancet. 2009;373(9669):1097-104.

34. Eisenberg BL, Harris J, Blanke CD, Demetri GD, Heinrich MC, Watson JC, et al. Phase II trial of neoadjuvant/adjuvant imatinib mesylate (IM) for advanced primary and metastatic/recurrent operable gastrointestinal stromal tumor (GIST): early results of RTOG 0132/ACRIN 6665. J Surg Oncol. 2009;99(1):42-7.

35. Demetri GD, von Mehren M, Blanke CD, Van den Abbeele AD, Eisenberg B, Roberts PJ, et al. Efficacy and safety of imatinib mesylate in advanced gastrointestinal stromal tumors. N Engl J Med. 2002;347(7):472-80.

36. Fernández JA, Parrilla P. Surgical treatment of an advanced GIST the age of imatinib. Cir Esp. 2009;86(1):3-12.

37. Gold JS, Dematteo RP. Combined surgical and molecular therapy: the gastrointestinal stromal tumor model. Ann Surg. 2006;244(2):176-84.

Manuscrito recibido el 10 de mayo de 2010 y aceptado para publicación el 10 de junio de 2010.

\section{Correspondencia:}

Rolando Mendívil Zapata

Av. Arnaldo Marquez 653.

Lima 11, perú.

Correo-e: rolandomendivil@hotmail.com

José Cabanillas Lapa

Correo-e: josel_cabanillas@hotmail.com 